\title{
Antioxidant Status of Children Infected with Plasmodium falciparum Malaria in Kebbi Metropolis, Northwestern Nigeria
}

\author{
Mode Muhammad Abduljalil ${ }^{1 *}$, Mu'azu Gusau Abubakar ${ }^{2}$ and Muhammad Abdulmalik Danjuma \\ ${ }^{1}$ Central Advance Science Laboratory Complex, Usmanu Danfodiyo University, Sokoto, Nigeria \\ ${ }^{2}$ Department of Biochemistry, Usmanu Danfodiyo University, Sokoto, Nigeria \\ *Corresponding author: Mode Muhammad Abduljalil, Central Advance Science Laboratory Complex, \\ Usmanu Danfodiyo University, Sokoto, Nigeria, Tel: +234-(0)-8032561547
}

\begin{abstract}
Malaria is a global life aggressive disease caused by the Plasmodium parasite to a host after infected anopheles mosquito leading to release of free radicals which have the capacity to induce oxidative stress. This study was carried out to assess the effect of malaria parasite (Plasmodium falciparum) on some antioxidants (vitamins $A, C, E$ and reduced glutathione) and lipid peroxidation marker (MDA) in children attending Sir Yahaya Memorial Hospital, Birnin Kebbi, Kebbi State, Nigeria. Blood samples were collected from untreated subjects upon confirmation of Plasmodium falciparum parasitaemia using the Rapid test kit (SD Bioline Malaria Ag P.f) method. One hundred and twelve consenting subjects (72 positive and 40 negative subjects) comprising of both sexes were randomly selected. Vitamin A was determined using a method of Bassey, et al. [1] while vitamins $C$ and $E$ using a method of Baker and Frank [2]. Reduced glutathione and MDA were determined using methods of Patterson and Lazarow [3] and Abubakar, et al. [4] respectively. Results were analysed using SPSS version 16.0 and significance between groups was ascertained using students' T-test. Result showed that level of antioxidant vitamins (vitamins $\mathrm{A}, \mathrm{C}, \& \mathrm{E}$ ) and reduced glutathione $(\mathrm{GSH})$ in malarial positive subjects were significantly lower $(p<0.05)$ compared to control subjects. Similarly, lipid peroxidation marker (MDA) were significantly $(p<0.05)$ higher in children with parasitaemia than in non-parasitaemia controls. The decrease in the levels of antioxidant vitamins ( $\mathrm{A}, \mathrm{C}$ and $\mathrm{E}$ ) and reduced glutathione (GSH) as observed may be due toneed of antioxidants to scavenge the free radicals caused by malarial infection.
\end{abstract}

\section{Keywords}

Antioxidant markers, Children, Plasmodium falciparum, Lipid peroxidation

\section{Introduction}

Malaria is considered as one of the major life threatening disease in developing countries, despite numerous concerted efforts by the governments of various countries and other donor organizations including several drugs/combinations developed acting against the parasite [5]. Recent world malaria report released in 2020 by WHO showed that in 2019 alone, a global estimation of 229 million cases of infection and 409,000 deaths were recorded with children under the age of 5 years been the most vulnerable group as they accounted for $274,000(67 \%)$ of all the malaria deaths worldwide [6]. The WHO's report also revealed that Africa has high portion of the global malaria burden because in 2019 only, the region has an estimation of $94 \%$ of all malaria cases and death. Six (6) countries were reported to be accounted for approximately half of all global malaria death with Nigeria having the highest figure of $23 \%$ followed by Democratic Republic of Congo with 11\% [6]. In Nigeria, malaria is transmitted all over the country with about $76 \%$ of the populace live in high transmission areas whereas $24 \%$ of the population live in low transmission areas [7]. In southern part of Nigeria, the malaria transmission can last all over the year while in northern part is just about 3 to 5 months. Wassmer and Grau [8] revealed that malaria is an olden menace to human health and is one of the main causes of illness and death globally. There are five (5) human malarial species: Plasmodium

Citation: Abduljalil MM, Abubakar MG, Danjuma MA (2021) Antioxidant Status of Children Infected with Plasmodium falciparum Malaria in Kebbi Metropolis, Northwestern Nigeria. Int J Trop Dis 4:053. doi.org/10.23937/2643-461X/1710053

Accepted: October 18, 2021; Published: October 20, 2021

Copyright: (C) 2021 Abduljalil MM, et al. This is an open-access article distributed under the terms of the Creative Commons Attribution License, which permits unrestricted use, distribution, and reproduction in any medium, provided the original author and source are credited. 
falciparum, malariae, ovale, vivax and $P$. Knowlesi where Plasmodium falciparum is the most virulent [9]. Most of the disease caused by infection with Plasmodium spp. is caused by $P$. falciparum [10] and it is considered as the deadliest form, it can cause acute anaemia through red blood cell destruction $[9,11,12]$. Most of malaria cases admitted to hospitals are due to $P$. falciparum of which children less than 5 years of age and pregnant women are most affected $[13,14]$. Babalola, et al. [9] reported that the mayhem triggered by $P$. falciparum malaria in Africa cannot be overemphasized.

When an infected female of anopheles mosquito bite a vertebrate host (e.g. human), the disease is triggered. In humans, an asexual multiplicative and evolutionary cycle takes place, initially in the hepatocytes and subsequently intra erythrocytes. In each intra erythrocyte cycle, merozoites are released which continuously invading other red cells in successive cycles, leading to a more or less severe anemia according to the infecting Plasmodium species cycle [15-17].

Frequently, in a first phase, an infected individual develops unspecific symptoms, which can be confused with any flu syndrome which includes fever, rigors, and chills [18]. Young children with severe malaria may develop a serious clinical picture with severe anaemia, extreme weakness, impaired consciousness, respiratory distress, convulsions, and hypoglycaemia [16]. Severe anemia in such cases plays a substantial part in its morbidity and mortality [19]. Malarial infection are reported to increase the production of reactive oxygen species (ROS) which may lead to erythrocyte membrane damage and contribute towards the anaemia in the host [9]. Studies by Ogbodo, et al. [20]; Atiku, et al. [21] and Babalola, et al. [9] disclosed that increased oxidative stress in patients infected with $P$. falciparum malaria infection is related to severity of disease and anaemia. Furthermore, Narsaria, et al. [22] and Anton Gotz, et al. [23] reported that malaria infection triggers the immune system of the body followed by the release of reactive oxygen species (ROS) such as superoxide radicals, hydrogen peroxide, hydroxyl radical, lipid peroxides and other related species through engaged activated monocytes and neutrophils as an antimicrobial action. Also malaria parasite activated certain cells in the provision of ROS leading haemoglobin degradation [23]. It has been reported that oxidative stress plays vital role in the progression of malarial anaemia and Plasmodium falciparum infected red cells produce more reactive oxygen species [24]. Despite the action of oxidative stress during malaria infection is still not elucidated, some scientists revealed a defensive role, while others confirmed a relation with malarial pathology [25].

In normal situations, there is a balance between the production of free radicals (reactive oxygen species) and defences of antioxidants production, so the interruption of this balance is responsible for the cell damage, which is termed oxidative stress $[26,27]$. This happens when the excessive production of oxygen radical in the cell overwhelm the normal capacity of antioxidants $[26,28]$. These antioxidants molecules have the ability to carefully interact with free radicals to dismiss their harmful effect before important biological molecules are impaired. Oxidative stress is a detrimental process which occurs when excessive free radicals engulf antioxidants a condition that undesirably affect several cellular structures such as membrane lipids, proteins, lipoproteins, and deoxyribonucleic acid [29]. Francois [28] further reported that if the control of ROS failed by internal defence mechanism, this could lead to toxicity such as genotoxicity, cytotoxicity, and even when mutated cells can proliferate i.e. carcinogenesis.

Moreover, human body possesses certain mechanisms to diminish the cellular effects of reactive oxygen species perhaps through nutritional diet, which are considered as the major source of antioxidants or can be synthesized in the body through many intracellular mechanisms [30]. Also, Onyesom, et al. [30] reported that uric acid, superoxide dismutase, vitamin $\mathrm{E}$ restrain the damaging phase of reactive oxygen species, while glutathione peroxidase, vitamins $A$ and C, catalase, restrain the initial production of reactive oxygen species. It has been further reported that serum antioxidant vitamins (Vitamins A, C and E) are important markers of oxidative stress and demonstrated to scavenge the radicals imposed by malaria infection [31].

An oxidative stress marker: malondialdehyde (MDA) levels in persons with malaria are shown to be higher when compared to the uninfected ones, while antioxidant enzyme catalase activity are lower in malaria infected patients compared to negative control [32]. Also, Cabrales, et al. [33] disclosed that increased production of free radicals which will lead to oxidative stress will subsequently lead to increased formation of malondialdehyde (MDA) which is an important lipid peroxidation marker.

Several studies in some part of the nation have reported that malaria infection inflicts remarkable oxidative stress on the host. However there is scarce data from Kebbi State, though there is disparity due to factors such as race, socio-economic and certain environmental or ecological factors. Hence the need to investigate the levels of serum antioxidant vitamins (Vitamins A, C and E) and endogenous antioxidants such as reduced glutathione (GSH) and lipid peroxidation marker (MDA) in malaria infected children in Kebbi State metropolis to determine if the impact of these factors may alter the already established findingsas reported in a similar study by Abubakar, et al. [11]. Therefore, the aim of this study is to assess the levels of these important antioxidants (Vitamins A, C, E and GSH) and lipid peroxidation marker (MDA) in children infected with Plasmodium falciparum of Kebbi State metropolis. 


\section{Materials and Methods}

\section{Research location}

The research was conducted in Birnin Kebbi metropolis, Kebbi State, North-western Nigeria. Samples were collected from Sir Yahaya Memorial Hospital, Kebbi State. Kebbi State has a total population of 3,238,628 based on 2006 National census and is located on the north western part of Nigeria. It has a total landmass of about 37,699 square kilometres out of which $36.46 \%$ is made up of farmland. However, about one third of the state is situated in desert prone environment thus making it one of the front-line states for the menace of drought and desertification. The state shares an extensive border with Niger Republic to the North and Benin Republic to the west with many intercultural and ethnic linkages.

\section{Sample size}

Simple random sampling technique was used in selecting the participants of the study. The random selection was used to guarantee that each individual has an independent and equal chance of being selected. The method is also very fair and unbiased.

Research and Ethics Committee of Usmanu Danfodiyo University have approved all the experimental protocols via a protocol number UDUS/UREC/2019/018 prior to the commencement of the study. Parents of the children enrolled were informed using a standard informed consent form.

A total of One Hundred and Twelve (112) subjects comprising seventy two (72) malarial positive subjects between the ages of 0 months to 6 years and fourty (40) apparently healthy non-malaria volunteers (control) subjects (age and gender mismatched) were enrolled into the study. The research is a case control study involving Plasmodium falciparum malaria parasitized children (subjects) and non-malaria parasitized control subjects.

Inclusion criteria: Children that met the inclusion criteria are children aged between 0 to 6 years who are tested positive with Plasmodium falciparum parasitaemia and are visiting the Sir Yahaya Memorial Hospital Kebbi State, Nigeria. Furthermore, only children whose parents and guardian gave a written informed consent for their ward are included in this study.

Exclusion criteria: Children aged above 6 years and also whose parents or guardian refused to give a written informed consent for their ward to participate in this study were excluded from the study. Additionally, children reported to have transfused with blood in the last Three (3) months or are on antioxidants supplementation were also excluded.

\section{Sample collection and sample treatments}

Upon acceptance to participate in the study, a blood sample was collected from each patient using multi sample needle, with EDTA vacutainers and plain vacutainers specimen bottle. Plain vacutainers were centrifuged for five minutes at $3000 \mathrm{rpm}$ and used for biochemical analysis. The presence of Plasmodium falciparum malaria was determined with rapid diagnostic test (SD Bioline Malaria Ag P.f) using a blood sample in EDTA vacutainer.

\section{Methodology}

All the reagents and chemicals used for the analysis were of analytical grade. Method as described by Bassey, et al. [1] has been followed for analysis of vitamin Awhile a method of Baker and Frank [2] was employed for determination of vitamins $\mathrm{C}$ and $\mathrm{E}$. Reduced glutathione was measured in blood serum using a method of Patterson and Lazarow [3] whereas lipid peroxidation marker (MDA) was measured using the thiobarbituric acid reactions (TBARS) as reported by Abubakar, et al. [4].

\section{Statistical analysis}

Result of the study are presented as Mean \pm SEM. Statistical significance between groups were assessed using students' T-test and statistical significance was established at $P<0.05$. The SPSS package version 16 and InStat 3 was used for the analysis as described [34].

\section{Results and Discussion}

Demographic and clinical characteristic of the study subjects are presented in Table 1. For all the infected subjects enrolled into the study, male are the majority with 40 subjects representing $55.56 \%$, while the remaining $32(44.44 \%)$ are female. Likewise, majority of the control subjects (uninfected) enrolled are male who constituted 23 subjects (57.5\%), while the remaining 17 subjects $(42.5 \%)$ are female. Furthermore, majority of the malaria infected and uninfected (control) subjects included in this study were aged between 5 to 6 years, representing $47.22 \%$ and $47.5 \%$ respectively, followed by those within the age of 3 to 4 years representing $31.95 \%$ and $32.5 \%$ for study (infected) subjects and control group respectively. The remaining subjects in

Table 1: Demographic characteristics of the children with Plasmodium falciparum malaria.

\begin{tabular}{|l|l|l|}
\hline $\begin{array}{l}\text { Characteristics } \\
(\mathbf{n}=\mathbf{7 2})\end{array}$ & $\begin{array}{l}\text { Infected subjects } \\
(\mathbf{n}=\mathbf{4 0})\end{array}$ & $\begin{array}{l}\text { Control } \\
\text { subjects }\end{array}$ \\
\hline Gender & $40(55.56)$ & $23(57.5)$ \\
\hline Male & $32(44.44)$ & $17(42.5)$ \\
\hline Female & & \\
\hline Age (years) & $15(20.83)$ & $08(20.0)$ \\
\hline $0-2$ & $23(31.95)$ & $13(32.5)$ \\
\hline $3-4$ & $34(47.22)$ & $19(47.5)$ \\
\hline $5-6$ & & \\
\hline
\end{tabular}

$\mathrm{N}$ : Percentage; $\mathrm{n}$ : number of sample 
Table 2: Effect of Plasmodium falciparum malaria on some antioxidants parameters of the study subjects.

\begin{tabular}{|l|l|l|}
\hline Antioxidants & $\begin{array}{l}\text { Infected subjects } \\
(\mathbf{n = 7 2})\end{array}$ & $\begin{array}{l}\text { Control subjects } \\
\mathbf{( n = 4 0 )}\end{array}$ \\
\hline Vitamin A (mg/dl) & $80.384 \pm 1.391^{*}$ & $94.609 \pm 1.161^{*}$ \\
\hline Vitamin C (mg/dl) & $81.505 \pm 1.844^{*}$ & $97.038 \pm 1.360^{*}$ \\
\hline Vitamin E (mg/dl) & $79.847 \pm 2.074^{*}$ & $95.892 \pm 0.822^{*}$ \\
\hline GSH $(\mathrm{mg} / \mathrm{dl})$ & $42.540 \pm 1.103^{*}$ & $56.442 \pm 1.856^{*}$ \\
\hline MDA $(\mathrm{nmol} / \mathrm{L})$ & $68.901 \pm 4.819^{*}$ & $43.553 \pm 5.059^{*}$ \\
\hline
\end{tabular}

$\mathrm{n}$ : number of sample, GSH: Reduced Glutathione, MDA: Malondialdehyde

Values are expressed as Mean \pm SEM. Values with the same superscript in the same row are significantly different $(P<0.05)$.

both the two (2) groups were aged between 0 to 2 years.

The result of the study as presented in Table 2, showed that malarial infection significantly $(P<0.05)$ decreases serum levels of antioxidant vitamins: $A, C$ and Eand Reduced Glutathione (GSH) when compared with the non-infected subjects. Likewise, Malondialdehyde (MDA) levels was significantly higher $(P<0.05)$ in malaria infected children, in comparison with the control group.

The result presented in Table 2 showed that Plasmodium falciparum malarial infection significantly $(P<0.05)$ lower the serum levels of antioxidant vitamins $A, C$, and $E$ as well as reduced glutathione (GSH) and significantly increases lipid peroxidation marker (MDA). It has been confirmed that Plasmodium falciparum merozoites as an antigens triggers the production of reactive oxygen species (ROS) by polymorpho nuclear and mononuclear leukocytes [35]. Malaria infection triggers the generation of hydroxyl radicals $(\mathrm{OH} \bullet)$ in the liver, hence may be the foremost reason for the induction of oxidative stress and apoptosis [36]. Furthermore, it has been reported that infection of erythrocytes by $P$. falciparum produces hydroxyl group $(\mathrm{OH} \bullet)$ radicals and hydrogen peroxide $\left(\mathrm{H}_{2} \mathrm{O}_{2}\right)$ about doubled when compared to normal erythrocytes [37]. Nitric oxide (NO) is an innate immune mediator and another free radical species that are involved in progression of malaria infection though its role is still deliberated $[33,38]$. Oxidative stress in children infected with $P$. falciparum is recognised to induce anaemia via a chain of processes and the main target of oxidative stress is the red blood cell due to their principal function as $\mathrm{O}_{2}$ carrying cells [39]. Several scientists proposed that cerebral malaria may be perhaps an ill-fated consequence of high amounts of nitric oxide (NO) production, in order to stimulate the death of the parasites $[40,41]$ though other group of scientist believed that cerebral malaria results from a low bioavailability of nitric oxide [42]. Another possible cause of free radical in malaria infected subjects is the human haemoglobin molecule, which serves as the best source of amino acid to the malaria parasite particularly during erythrocytic stage of the infection, thereby leading to release of significant amount of circulating heme, where $\mathrm{Fe}^{2+}$ is oxidized to form $\mathrm{Fe}^{3+}$ and the electrons produced during this process react with molecular oxygen to form ROS [43] and capable of inducing intravascular oxidative stress and resulting to changes in erythrocytes and endothelial cells, which will help the internalization of the malaria parasite in liver and brain [44]. Moreover, reactive oxygen species (ROS) are also generated in the mitochondria through various metabolic processes [22,45-48]. These free radical productions are triggered by malaria parasite which will subsequently lead to an antioxidant defence in the host cell to halt the infection [49-51]. Guha, et al. [52] and Sohail, et al. [51] reported higher levels of oxidative stress markers in malaria infected humans compared to uninfected subjects. Numerous studies have reported oxidative stress, hypoxia, increased inflammation, and hepatocyte apoptosis in malaria-infected livers [53]. Oxidative stress is considered as a main factor in the pathogenesis of malaria and contributes to the severity of malaria related complications [26].

Antioxidant molecules such as vitamins A, C, E and reduced glutathione (GSH) are very important in scavenging the free radicals released as a result of malaria infection. Reduced glutathione (GSH) molecule is reported to be the best influential protector of eukaryotic cells in the host defence against oxidative stress $[37,54]$. Malondialdehyde is a product of lipid peroxidation and hence recommended as significant biomarker of oxidative stress in both severe malaria and placental malarial [37,55]. Meanwhile, several documented evidences showed that malaria infection results in increased ROS as well as reduction of antioxidants, therefore oxidative stress in malaria infected patients is distinct as the disparity between the two can cause either ROS excess or antioxidant decline [9]. Increase in lipid peroxidation marker (MDA) level and decrease in antioxidants vitamins (vitamins $\mathrm{A}, \mathrm{C}$ and $\mathrm{E}$ ) have been reported to be responsible for the progress of oxidative stress in malaria patients $[9,11,56]$. Human biological system possesses a number of defence mechanisms against reactive oxygen species, which include the synthesis and utilization of antioxidants $[57,58]$ as these reactive oxygen species has the ability to induce lipid peroxidation and cell damage [11]. Therefore, the effect of reactive oxygen species could lead to significant reduction of antioxidant levels as showed in this study. Furthermore, this strengthens the crucial role played by antioxidants in the defence of deleterious biological consequences induced ROS. Reduced glutathione (GSH) is an extremely important cell protectant as it directly quenches reactive hydroxyl free radicals, other oxygen centred free radicals, and radical centres on DNA and other biomolecules [54]. Vitamin C or Ascorbic acid $(\mathrm{AH}-)$ is a water soluble vitamin and a potent reducing agent required by the biological system to scavenge several radical species [59]. Lipid peroxidation (LPO) radical can damage the macromolecules (DNA, RNA, 
and proteins) and can initiate cytotoxic, genotoxic, and inflammatory reactions. Vitamin $C$ converts lipid peroxidation products into unreactive vitamin C-LPO products and helps to prevent the interaction of macromolecules (DNA, RNA, and proteins) with LPO• radicals [60]. Likewise, vitamin $C$ is reported to increases iron absorption by reducing $\mathrm{Fe}^{3+}$ to $\mathrm{Fe}^{2+}$ [61]. Vitamins $A$ and $E$ are lipid soluble antioxidants that possess the ability to directly quench free radicals and function as a membrane stabilizer [62] and several studies have shown that vitamin A is effective antioxidant [59].

The decreased antioxidant concentrations of vitamins A, C, and E observed in this study may be due to their increased utilization by the body to solve the deleterious effects of free radicals generated by parasitaemia [37] as highlighted above. Furthermore, the decrease in the level of reduced glutathione (GSH) experienced may be due to its over utilization to directly quenches the devastating hydroxyl free radicals produced by the body as a result of the parasite infection [56]. Likewise, increase in MDA observed in this study may be due to increase in peroxidation of membrane lipids caused by the parasite [63] or could be due to decreased activity of the defence system protecting tissues from free radical damage [64]. Results of this study showed an inverse relationship between antioxidant levels and lipid peroxidation. The result of the study is and are consistent with the earlier studies by Onyesom, et al. [30] and Abubakar, et al. [11] who reported a decrease in the antioxidants vitamins, increased in the levels of MDA and GSH. Guha, et al. [52]; Kulkarni, et al. [65] also reported an imbalance in the oxidants and antioxidants levels in the serum of malarial infected patients which also confirmed to the finding of this study. Similarly, the finding of our study is in conformity with the study carried out by Idonije, et al. [63] who reported decreased antioxidants capacity in malaria infected patients especially $P$. falciparum compared to uninfected subject. The finding of his study [63] directed him to conclude that there is oxidative stress in children with parasitaemia due to significantly higher levels of lipid peroxidation andreduced glutathione he observed. Studies by Egwunyenga, et al. [66] and Abubakar, et al. [11] revealed a significant decrease in the level of vitamin $C$ which also coincided with increase in the level of MDA in P. falciparum infected children compared to control subjects. Additionally, studies by Sobolewski, et al. [67]; Guha, et al. [52] and Sohail, et al. [51] reported a significant increase in oxidative stress markers in malaria infected humans compared to uninfected controls. Equally, Egwunyenga, et al. [66]; Atiku, et al. [21] also reported higher level of lipid peroxidation marker (MDA) in malaria infected patients than uninfected control subjects. Moreover, a recent studies conducted by Atiku, et al. [21] and Babalola, et al. [9] showed an increase in MDA (lipid peroxidation marker) and decrease in antioxidants
Vitamin C and GSH in malaria patients compared to uninfected subjects. Likewise, another Ethiopian study carried out by Ebrahim, et al. [68] showed that the total oxidative status in malaria patients was significantly higher than that in control subjects. Consequently, the Plasmodium falciparum virulence appears to depend basically on the patients' antioxidant capacities and this is also determined by the concentrations of antioxidant micronutrients [20]. Nevertheless, biological system possesses several defence mechanisms to reduce the cellular effects of ROS triggered by $P$. falciparum such as intracellular synthesis of antioxidants or acquired nutritionally through diet.

\section{Conclusion}

This finding may have added more information to the sparse reports (if any) on changes in antioxidant profile of malaria infected children living in Kebbi metropolis, Kebbi State, North-western Nigeria. The finding of this study maysuggest that exogenous micronutrient with antioxidant vitamins ( $A, C$ and $E$ ) may improve the management of children infected with $P$. falciparum malaria regardless of sex.

\section{Conflict of Interest}

The authors have declared no conflict of interest and no other relationship or activities that could appear to have influenced this study. Authors have not received any financial support from any organization and no financial relationship with any organization or individual that might have an interest in the previous three (3) years.

\section{Acknowledgement}

The authors are grateful to the management of Sir Yahaya Memorial Hospital, Birnin-Kebbi, Kebbi State particularly staff of Microbiology Laboratory for their support throughout the study. The authors also acknowledged the technical support received from the staff of Biochemistry Laboratory, Usmanu Danfodiyo University, Sokoto State, Nigeria.

\section{References}

1. Bassey OA, Lowry OH, Brock MJ, Lopez JA (1946) The determination of vitamin $A$ and carotene in small quantities of blood serum. J Biol Chem 166: 177-188.

2. Baker H, Frank O (1968) Clinical Vitaminology: Method and interpretation. ( $1^{\text {st }}$ edn), Interscience publishers, New York, 228-229.

3. Patterson JW, Lazarow A (1955) Methods of Biochemical Analysis. ( $3^{\text {rd }}$ edn), Inter Science, New York, 251-259.

4. Abubakar MG, Taylor A, Ferns GA (2002) Regional distribution of Aluminium in the rat brain. Influence of Vitamin E metals. Lons Biol Med 7: 217-221.

5. Kumar S, Mina PR, Kumar R, Pal A, Ahmad A, et al. (2021) 4-chlorothymol exerts antiplasmodial activity impeding redox defense system in Plasmodium falciparum. Front Pharmacol 12: 628970. 
6. World Health Organization (WHO) (2021) World malaria report 2019.

7. USAID (2020) President's Malaria Initiative Nigeria Malaria Operational Plan FY 2020.

8. Wassmer CS, Grau GER (2017) Severe malaria: What's new on the pathogenesis front? Int J Parasitol 47: 145-152.

9. Babalola AS, Jonathan J, Michael BE (2020) Oxidative stress and anti-oxidants in asymptomatic malaria-positive patients: A hospital-based cross-sectional Nigerian study. Egypt J Intern Med 32: 23.

10. World Health Organization (2017) World malaria report 2017. Geneva.

11. Abubakar MG, Usman SM, Dandare SU (2016) Oxidant status of children infected with Plasmodium falciparum malaria in katsina metropolis, North Western Nigeria. Afr J Infect Dis 10: 17-20.

12. White NJ (2018) Anaemia and malaria. Malar J 17: 371.

13. Kavishe RA, Koenderink JB, Alifrangis M (2017) Oxidative stress in malaria and artemisinin combination therapy: Pros and Cons. FEBS J 284: 2579-2591.

14. Abossie A, Yohanes T, Nedu A, Tafesse W, Damitie M (2020) Prevalence of malaria and associated risk factors among febrile children under five years: A cross-sectional study in arba minch zuria district, South Ethiopia. Infect Drug Resist 13: 363-372.

15. Collins CR, Hackett F, Strath M, Penzo M, Withers-Martinez C, et al. (2013) Malaria parasite cGMP-dependent protein kinase regulates blood stage merozoite secretory organelle discharge and egress. PLoS Pathog 9: e1003344.

16. Wockner LF, Hoffmann I, Webb L, Mordmüller B, Murphy SC, et al. (2020) Growth rate of Plasmodium falciparum: Analysis of parasite growth data from malaria volunteer infection studies. J Infect Dis 221: 963-972.

17. Venugopal K, Hentzschel F, Valkiūnas G, Marti M (2020) Plasmodium asexual growth and sexual development in the haematopoietic niche of the host. Nat Rev Microbiol 18: 177-189.

18. Morakinyo OM, Balogun FM, Fagbamigbe AF (2018) Housing type and risk of malaria among under-five children in Nigeria: Evidence from the malaria indicator survey. Malar J 17: 311.

19. Scheu K, Adegnika AA, Addo MM, Ansong D, Cramer JP, et al. (2019) Determinants of post-malarial anemia in African children treated with parenteral artesunate. Sci Rep 9: 18134

20. Ogbodo SO, Okaka ANC, Nwagha UI, Ejezie F, Okafor C (2014) Oxidative stress in symptomatic malaria parasitemic pregnant women from malaria endemic area of Nigeria. Am J Med Med Sci 4: 168-174.

21. Atiku SM, Louise N, Kasozi DM (2019) Severe oxidative stress in sickle cell disease patients with uncomplicated Plasmodium falciparum malaria in Kampala, Uganda. BMC Infect Dis 19: 600.

22. Narsaria N, Mohanty C, Das BK, Mishra SP, Prasad R (2012) Oxidative stress in children with severe malaria. J Trop Pediatr 58: 147-150.

23. Gotz A, Ty MC, Rodriguez A (2019) Oxidative stress enhances dendritic cell responses to Plasmodium falciparum. Immunohorizons 3: 511-518.

24. Chandra P, D'Souza V, D'Souza B (2006) Comparative study on lipid peroxidation and antioxidant vitamins $E$ and
$\mathrm{C}$ in falciparum and Vivax malaria. Ind $\mathrm{J}$ Clin Biochem 21: 103-106.

25. Pabon A, Carmona J, Burgos LC, Blair S (2003) Oxidative stress in patients with non-complicated malaria. Clin Biochem 36: 71-78.

26. Sarr D, Cooper CA, Bracken TC, Martinez-Uribe O, Nagy T, et al. (2017) Oxidative stress: A potential therapeutic target in placental malaria. Immunohorizons 1: 29-41.

27. Pizzino G, Irrera N, Cucinotta M, Pallio G, Mannino F, et al. (2017) Oxidative stress: Harms and benefits for human health. Oxid Med Cell Longev 2017: 8416763.

28. Gagné F, (2014) Oxidative stress. In: Francois Gagné, Biochemical Ecotoxicology. Chapter 6, Academic Press.

29. Lobo V, Patil A, Phatak A, Chandra N (2010) Free radicals, antioxidants and functional foods: Impact on human health. Pharmacogn Rev 4: 118-126.

30. Onyesom I, Ejovi O, Omoghene O (2012) Total antioxidant capacity in serum of Plasmodium falciparum malarial infected patients receiving artemisinin-based combination Therapy. American Journal of Medicine and Medical Sciences 2: 1-3.

31. Onyesom I, Ekeanyanwu RC, Achuka N (2010) Correlation between moderate Plasmodium falciparum malarial parasitaemia and antioxidant vitamins in serum of infected children in South Eastern Nigeria. Afr J Biochem Res 4: 261-264.

32. Sakyi SA, Ephraim RKD, Antoh EO, Obirikorang C, Berchie GO (2012) Lipid peroxidation and catalase levels among children presenting with severe falciparum malaria in the Sefwi Wiawso Municipality, Ghana. J Med Sci 12: 141-147.

33. Cabrales P, Zanini GM, Meays D, Frangos JA, Carvalho LJM (2011) Nitric Oxide protection against murine cerebral malaria is associated with improved cerebral microcirculatory physiology. J Infect Dis 203: 1454-1463.

34. Ogbeibu AE (2005) Biostatistics: A practical approach to research and data handling. Mindox Publishing Ltd., Nigeria, 20.

35. Aitken EH, Alemu A, Rogerson SJ (2018) Neutrophils and Malaria. Front Immunol 9: 3005.

36. Renia L, Goh YS (2016) Malaria Parasites: The great escape. Front Immunol 7: 463.

37. Percario S, Moreira DR, Gomes BA, Ferreira ME, Goncalves AC, et al. (2012) Oxidative stress in malaria. Int J Mol Sci 13: $16346-16372$.

38. Corbett Y, D'Alessandro S, Parapini S, Scaccabarozzi D, Kalantari P (2018) Interplay between Plasmodium falciparum haemozoin and L-arginine: Implication for nitric oxide production. Malar J 17: 456.

39. Bissinger R, Bhuyan AA, Qadri SM, Lang F (2019) Oxidative stress, eryptosis and anemia: A pivotal mechanistic nexus in systemic diseases. FEBS J 286: 826-854.

40. Maneerat $Y$, Viriyavejakul $P$, Punpoowong $B$, Jones $M$, Wilairatana $P$, et al. (2000) Inducible nitric oxide synthase expression is increased in the brain in fatal cerebral malaria. Histopathology 37: 269-277.

41. Idro R, Marsh K, John CC, Newton CRJ (2010) Cerebral malaria: Mechanisms of brain injury and strategies for improved neurocognitive outcome. Pediatr Res 68: 267274.

42. Zheng Z, Liu H, Wang X, Zhang Y, Qu S, et al. (2021) Artesunate and Tetramethylpyrazine exert effects on 
experimental cerebral malaria in a mechanism of protein S-Nitrosylation, ACS Infect Dis 7: 2836-2849.

43. Deshmukh R, Trivedi V (2013) Pro-stimulatory role of methemoglobin in inflammation through hemin oxidation and polymerization. Inflamm Allergy Drug Targets 12: 6878.

44. Kumar S, Bandyopadhyay U (2005) Free heme toxicity and its detoxification systems in human. Toxicol Lett 157: 175188.

45. Trivedi V, Chand P, Srivastava K, Puri SK, Maulik PR, et al. (2005) Clotrimazole inhibits hemoperoxidase of Plasmodium falciparum and induces oxidative stress. Proposed antimalarial mechanism of clotrimazole. J Biol Chem 280: 41129-41136.

46. Van Dooren GG, Stimmler LM, McFadden GI (2006) Metabolic maps and functions of the Plasmodium mitochondrion. FEMS Microbiol Rev 30: 596-630.

47. Gomes BAQ (2011) Efeitos da Suplementação com Antioxidantes Sobre as Alterações Oxidativas Cerebrais e Pulmonaresem Malária Murina. Master's Thesis, Federal University of Para, Belem, PA, Brazil.

48. Silva LD (2011) Efeito da Suplementação com Antioxidantes Sobre as Alterações Oxidativas e Produção de Interferon Gamma e Fator de Necrose Tumoral Alfa emTecido Pulmonar de Camundongos Infectadospor Plasmodium Berghei. Master's Thesis, Federal University of Para, Belem, PA, Brazil.

49. Keller CC, Kremsner PG, Hittner JB, Misukonis MA, Weinberg JB, et al. (2004) Elevated nitric oxide production in children with malarial anemia: Hemozoin-induced nitric oxide synthase type 2 transcripts and nitric oxide in blood mononuclear cells. Infect Immun 72: 4868-4873.

50. Potter SM, Mitchell AJ, Cowden WB, Sanni LA, Dinauer M, et al. (2005) Phagocyte-derived reactive oxygen species do not influence the progression of murine blood-stage malaria infections. Infect Immun 73: 4941-4947.

51. Sohail M, Kaul A, Raziuddin M, Adak T (2007) Decreased glutathione S-transferase activity: Diagnostic and protective role in vivax malaria. Clin Biochem 40: 377-382.

52. Guha M, Kumar S, Choubey V, Maity P, Bandyopadhyay U (2006) Apoptosis in liver during malaria: Role of oxidative stress and implication of mitochondrial pathway. FASEB $\mathrm{J}$ 20: 1224-1226.

53. Kim J, Wang S, Lee C, Sung S, Shin Y, et al. (2018) Bloodstage Plasmodium Berghei ANKA infection promotes hepatic fibrosis by enhancing hedgehog signaling in mice. Cell Physiol Biochem 50: 1414-1428.

54. Aquilano K, Baldelli S, Ciriolo MR (2014) Glutathione: New roles in redox signaling for an old antioxidant. Front Pharmacol 5: 196.

55. Megnekou R, Djontu JC, Bigoga JD, Medou FM, Tenou S, et al. (2015) Impact of placental Plasmodium falciparum malaria on the profile of some oxidative stress biomarkers in women living in Yaoundé, Cameroon. PLoS One 10: e0134633.

56. Oluba OM (2019) Erythrocyte lipid and antioxidant changes in Plasmodium falciparum-infected children attending mother and child hospital in Akure, Nigeria. Pak J Biol Sci 22: $257-264$.

57. Valko M, Leibfritz D, Moncol J, Cronin MTD, Mazur M, et al. (2007) Free radicals and antioxidants in normal physiological functions and human disease. Int $\mathrm{J}$ Biochem Cell Biol 39: 44-48.

58. He L, He T, Farrar S, Ji L, Liu T, et al. (2017) Antioxidants maintain cellular redox homeostasis by elimination of reactive oxygen species. Cell Physiol Biochem 44: 532553.

59. Fadime Eryılmaz Pehlivan (2017) Vitamin C: An antioxidant agent. Intech Open.

60. Hamza AH (2017) Vitamin C: An antioxidant agent. Publishing process manager, Edi Lipovic, 1-29.

61. Timoshnikov VA, Kobzeva TV, Polyakov NE, Kontoghiorghes GJ (2020) Redox interactions of Vitamin C and iron: Inhibition of the pro-oxidant activity by deferiprone. Int J Mol Sci 21: 3967.

62. Hamid NAA, Hasrul MA, Ruzanna RJ, Ibrahim IA, Baruah PS, et al. (2011) Effect of vitamin $E$ (Tri $E \AA)$ on antioxidant enzymes and DNA damage in rats following eight weeks exercise. Nutr J 10: 37.

63. Idonije OB, Festus O, Okhiai O, Akpamu U (2011) Comparative study of the status of a biomarker of lipid peroxidation (Malondialdehyde) in patients with Plasmodium falciparum and Plasmodium vivax Malaria Infection. Asian J Bio Sci 4: 506-513.

64. Bilgin R, Yalcin MS, Yucebilgic G, Koltas IS, Yazar S (2012) Oxidative stress in vivax malaria. Korean $\mathrm{J}$ Parasitol 50: 375-377.

65. Kulkarni AG, Suryakar AN, Sardeshmukh AS, Rathi DB (2003) Studies on biochemical changes with special reference to oxidant and antioxidants in malarial patients. Indian J Clin Biochem 18: 136-149.

66. Egwunyenga AO, Isamah G, Nmorsi OP (2004) Lipid peroxidation and ascorbic acid levels in Nigeria children with acute falciparum malaria. Afr J Biotechnol 3: 560-563.

67. Sobolewski $P$, Gramaglia I, Frangos JA, Intaglietta M, Heyde HVD (2005) Plasmodium berghei resists killing by reactive oxygen species. Infect Immun 73: 6704-6710.

68. Ebrahim A, Gnanasekaran N, Genet S (2019) Oxidative stress and diminished total antioxidant capacity in malaria patients correspond to increased parasitemia and severity of the disease. ROS 8: 287-296. 